\title{
Bipartite Regular Graphs with Fixed Diameter
}

\author{
H. J. Broersma and F. Gobel \\ Faculty of Applied Mathematics, University of Twente, P.O. Box 217, 7500 AE Enschede, \\ The Netherlands
}

For given nonnegative integers $k$ and $D$, we consider the problem of determining $n_{0}(k, D)$, the smallest number $n$ for which there exists a $k$-regular bipartite graph on $n$ vertices with diameter $D$. We solve the problem for all pairs $(k, D)$ with $D \neq 2(\bmod 4)$ and $D \neq 3(\bmod 4)$, for all pairs $(k, D)$ with $k$ even or $k$ prime and $D \neq 3(\bmod 4)$, for all pairs with $D \leq 9$ or $k \leq 4$, and for a few other pairs. In the remaining cases, we obtain lower and upper bounds for $n_{0}(k, D)$. (c) 1995 John Wiley \& Sons, Inc.

\section{INTRODUCTION}

We use Bondy and Murty [2] for terminology and notation not defined here and consider simple graphs only.

For given nonnegative integers $k$ and $D$, we consider the problem of determining $n_{0}(k, D)$, the smallest number $n$ for which there exists a $k$-regular bipartite graph on $n$ vertices with diameter $D$.

Bermond et al. [1] considered the following related problem: Given $\Delta, D$, find the largest number $n$ for which a graph on $n$ vertices exists with diameter $D$ and degrees at most $\Delta$. Delorme [4] considered the analogous problem for bipartite graphs, whereas Fellows et al. [5,6] considered planar graphs. Our research was triggered by Heydemann's question [7] whether a 4-regular graph on 14 vertices exists with diameter 2 . This question was answered affirmatively by Broersma and Jagers [3].

We solve the problem [of determining $n_{0}(k, D)$ ] for all pairs $(k, D)$ with $D \neq \equiv 2(\bmod 4)$ and $D \neq 3(\bmod 4)$, for all pairs $(k, D)$ with $k$ even or $k$ prime and $D \not \equiv 3$ $(\bmod 4)$, for all pairs with $D \leq 9$ or $k \leq 4$, and for a few other pairs. In the remaining cases, we obtain lower and upper bounds for $n_{0}(k, D)$.

$\mathrm{A}(k, D)$-graph is a $k$-regular bipartite graph with diameter $D$. Note that a $(k, D)$-graph exists if and only if $k=0, D=0$, or $k=1, D=1$, or $k \geq 2, D \geq 2$. In the sequel, we assume that $k \geq 2$ and $D \geq 2$.

\section{NOTATION AND PRELIMINARY RESULTS}

Let $G$ be a $(k, D)$-graph and let $v$ be a vertex of $G$ that has distance $D$ to some other vertex of $G$. Then, we denote the set of vertices at distance $i$ from $v$ in $G$ by $V_{i}$ and the cardinality of $V_{i}$ by $a_{i}(i=0,1, \ldots, D)$. Obviously, $a_{0}$ $=1$ and $a_{1}=k$. For $i=1,2, \ldots, D$, let $b_{i}$ be the number of edges of $G$ joining the vertices of $V_{i-1}$ and $V_{i}$. (Note that all $V_{i}$ are independent sets in $G$ since $G$ is bipartite.) Obviously, $b_{1}=k$ and $b_{2}=k(k-1)$.

The above notation will be used throughout this paper.

We start with some necessary conditions for $(k, D)$ graphs:

Lemma 1. If $G$ is $a(k, D)$-graph, then

(a) $a_{i-1}+a_{i+1} \geq k(i=1, \ldots, D-1)$,

(b) $b_{i-1}+b_{i}=k a_{i-1}(i=2, \ldots, D)$,

(c) $b_{i} \leq a_{i-1} a_{i}(i=1, \ldots, D)$,

(d) $\sum_{i=0}^{D}(-1)^{i} a_{i}=0$.

Proof.

(a) Since the $k$ neighbors of each vertex in $V_{i}$ are in $V_{i-1}$ $\cup V_{i+1}, a_{i-1}+a_{i+1} \geq k(i=1, \ldots, D-1)$.

(b) Since all vertices in $V_{i-1}$ have degree $k$ and are mutually nonadjacent, the definition of $b_{i-1}$ and $b_{i}$ implies that $b_{i-1}+b_{i}=k a_{i-1}(i=2, \ldots, D)$. 
(c) It is obvious from the definitions of $b_{i}$ and $a_{i}$ that $b_{i}$ $\leq a_{i-1} a_{i}(i=1, \ldots, D)$.

(d) Since $G$ is regular and bipartite with classes $V_{0} \cup V_{2}$ $\cup \cdots$ and $V_{1} \cup V_{3} \cup \cdots, \sum_{i=0}^{D}(-1)^{i} a_{i}=0$.

\section{Corollary 2.}

(a) All $b_{i}$ are divisible by $k$,

(b) $a_{i} \geq 2(i=1, \ldots, D-1)$.

Proof.

(a) Since $b_{1}=k$, and by Lemma 1(b), $b_{i}=k a_{i-1}-b_{i-1}$, all $b_{i}$ are divisible by $k$.

(b) If $a_{i}=1$ for some $i \in\{1, \ldots, D-1\}$, then by Lemma $1(\mathrm{~b}), b_{i}+b_{i+1}=k$, contradicting (a) since all $b_{i}$ are positive. Hence, $a_{i} \geq 2(i=1, \ldots, D$ $-1)$.

We denote $b_{i} / k$ by $f_{i}$ and note that all $f_{i}$ are integers by Corollary $2(\mathrm{~b})$. Moreover, we note that $a_{0}=f_{1}, a_{D}$ $=f_{D}$, and by Lemma $1(\mathrm{~b}), a_{i}=f_{i}+f_{i+1}(i=1, \ldots$, $D-1)$.

The notation $f_{i}$ will be used throughout this paper.

\section{Lemma 3.}

(a) $f_{i}+f_{i+1}+f_{i+2}+f_{i+3} \geq k(i=1, \ldots, D-3 ; D \geq 4)$,

(b) If $k$ is prime, then $f_{i}+f_{i+1}+f_{i+2}+f_{i+3} \geq k+1$ ( $i$ $=1, \ldots, D-3 ; D \geq 4)$.

(c) $f_{i}+f_{i+1}+f_{i+2} \geq\lceil 2 \sqrt{k}\rceil-1(i=1, \ldots, D-2 ; D$ $\geq 3$ ).

\section{Proof.}

(a) The inequality follows immediately from Lemma I (a).

(b) Suppose that $f_{i}+f_{i+1}+f_{i+2}+f_{i+3}=k$ for some $i$ $\in\{1, \ldots, D-3\}$. Then, $a_{i}=f_{i}+f_{i+1}<k$. Since $k$ is prime, this implies that $a_{i}$ is relative prime to $k$. By Lemma $1(\mathrm{c}), k f_{i+1} \leq a_{i} a_{i+1}$ and $k f_{i+2} \leq a_{i+1} a_{i+2}$, so that $k a_{i+1} \leq a_{i+1}\left(a_{i}+a_{i+2}\right)=a_{i+1} k$. This implies that $k f_{i+1}=a_{i} a_{i+1}$, or, equivalently, $a_{i+1}=\left[\left(k f_{i+1}\right) /\right.$ $a_{i}$ ]. Since $a_{i}$ is relative prime to $k$, we conclude that $a_{i}$ divides $f_{i+1}$, which is absurd since $0<f_{i+1}<a_{i}$. Hence, $f_{i}+f_{i+1}+f_{i+2}+f_{i+3} \geq k+1$.

(c) Suppose that $f_{i}+f_{i+1}+f_{i+2} \leq\lceil 2 \sqrt{k}\rceil-2$. Then, $f_{i}$ $+f_{i+2} \leq\lceil 2 \sqrt{k}\rceil-3$. We first show that

(1) $\left(f_{i}+f_{i+2}-k\right)^{2}-4 f_{i} f_{i+2}>0$.

Clearly, $\left(f_{i}+f_{i+2}-k\right)^{2}-4 f_{i} f_{i+2}=k^{2}-2 k\left(f_{i}\right.$ $\left.+f_{i+2}\right)+\left(f_{i}-f_{i+2}\right)^{2} \geq k(k-2\lceil 2 \sqrt{k}\rceil+6)$. From $(\sqrt{k}-2)^{2} \geqq 0$, we obtain $k-4 \sqrt{k}+4 \geq 0$ and $k+6 \geq 4 \sqrt{k}+2=2(2 \sqrt{k}+1)>2\lceil 2 \sqrt{k}$. Combining the above observations, we obtain (1).

By Lemma $1(\mathrm{c}), k f_{i+1} \leq\left(f_{i}+f_{i+1}\right)\left(f_{i+1}+f_{i+2}\right)$ or, equivalently, $f_{i+1}^{2}+\left(f_{i}+f_{i+2}-k\right) f_{i+1}$ $+f_{i} f_{i+2} \geq 0$; hence [using (1)] either
(2)

$$
\left.\left.k-\left(f_{i}+f_{i+2}\right)\right)+\sqrt{\left(f_{i}+f_{i+2}-k\right)^{2}-4 f_{i} f_{i+2}}\right] / 2
$$

or

(3) $\left[\left(k-\left(f_{i}+f_{i+2}\right)-\sqrt{\left(f_{i}+f_{i+2}-k\right)^{2}-4 f_{i} f_{i+2}}\right] / 2\right.$.

First suppose that (2) holds. Then, $4 \sqrt{k}-2$ $>2\lceil 2 \sqrt{k}\rceil-4 \geq 2\left(f_{i}+f_{i+1}+f_{i+2}\right) \geq k+f_{i}+f_{i+2}$ $+\sqrt{\left(f_{i}+f_{i+2}-k\right)^{2}-4 f_{i} f_{i+2}}>k+2$; hence, $(\sqrt{k}-2)^{2}=k-4 \sqrt{k}+4<0$, a contradiction.

Next suppose that $(3)$ holds. Since $f_{i+1} \geq 1$, from (3) we obtain $\sqrt{\left(f_{i}+f_{i+2}-k\right)^{2}-4 f_{i} f_{i+2}}$ $\leq k-\left(f_{i}+f_{i+2}\right)-2$, so that $\left(f_{i}+f_{i+2}-k\right)^{2}$ $-4 f_{i} f_{i+2} \leq\left(k-\left(f_{i}+f_{i+2}\right)\right)^{2}-4\left(k-\left(f_{i}+f_{i+2}\right)\right)$ +4 or, equivalently, $\left(f_{i}+1\right)\left(f_{i+2}+1\right) \geq k$. On the other hand, $f_{i}+f_{i+2} \leq\lceil 2 \sqrt{k}\rceil-3$ implies that $\left(f_{i}+1\right)+\left(f_{i+2}+1\right) \leq\lceil 2 \sqrt{k}\rceil-1<2 \sqrt{k}$; hence, $\left(f_{i}+1\right)\left(f_{i+2}+1\right)<k$, a contradiction.

We conclude that $f_{i}+f_{i+1}+f_{i+2} \geq\lceil 2 \sqrt{k}\rceil$ -1 .

We use the above results in the next section to determine lower bounds for $n_{0}(k, D)$. To determine upper bounds for $n_{0}(k, D)$ in the sequel, we often use the following lemma:

Lemma 4. Let $\alpha_{0}, \alpha_{1}, \ldots, \alpha_{D}$ and $\beta_{1}, \ldots, \beta_{D}$ be sequences of positive integers satisfying

(a) $\alpha_{0}=1, \alpha_{D}=1, \beta_{1}=k, \beta_{D}=k$ with $k \geq 2$,

(b) $\beta_{i}+\beta_{i+1}=k \alpha_{i}(i=1, \ldots, D-1)$,

(c) $\alpha_{i} \leq \beta_{i} \leq(k-1) \alpha_{i}(i=1, \ldots, D-1)$,

(d) $\beta_{i} \leq \alpha_{i-1} \alpha_{i}(i=1, \ldots, D)$.

Then, $a(k, D)$-graph on $\sum_{i=0}^{D} \alpha_{i}$ vertices exists.

Proof. Let $V_{0}, V_{1}, \ldots, V_{D}$ be pairwise disjoint vertex sets with $\left|V_{i}\right|=\alpha_{i}$. We will define a graph $G$ with vertex set $\cup_{i=0}^{D} V_{D}$ as follows:

For each edge $e$ of $G$, we will choose a number $i$ such that $e$ is incident with a vertex in $V_{i-1}$ and a vertex in $V_{i}$. This implies that $G$ is bipartite.

The indegree of a vertex in $V_{i}$ is defined as the number of its neighbors in $V_{i-1}$, and the outdegree, as the number of its neighbors in $V_{i+1}$. We will now prove the following claim by induction on $i$.

Claim. Between $V_{0}$ and $V_{1}, V_{1}$ and $V_{2}, \ldots, V_{i-1}$ and $V_{i}$, the edges of $G$ can be placed such that the degrees ( of the vertices) in $V_{0}, V_{1}, \ldots, V_{i-1}$ are $k$ and the indegrees for the vertices in $V_{j}$ differ by at most $1(j=1, \ldots, i)$; hence, the outdegrees also differ by at most $1(j=0, \ldots, i-1)$.

Proof of Claim. For $i=1$, the claim is true: By (c), $\alpha_{1} \leq \beta_{1}=k$, and by (d), $k=\beta_{1} \leq \alpha_{0} \alpha_{1}=\alpha_{1}$; hence, $\alpha_{1}$ $=k$. It is indeed possible to place the edges as required. 
Suppose that the claim is true for some $i$ with $1 \leq i$ $<D$. Between $V_{i-1}$ and $V_{i}$, there are $\beta_{i}$ edges [ this follows from (b)]; hence, the indegrees in $V_{i}$ can be determined as follows:

Write $\beta_{i}=q \alpha_{i}+r$ with $0 \leq r<\alpha_{i}$. Then, the indegrees in $V_{i}$ must be $r$ times $q+1$ and $\alpha_{i}-r$ times $q$. Now we can make all degrees in $V_{i}$ equal to $k$, and the outdegrees in $V_{i}$ will be $r$ times $k-q-1$ and $\alpha_{i}-r$ times $k-q$. The sum of these outdegrees is $r(k-q-1)+\left(\alpha_{i}-r\right)(k$ $-q)=(k-q) \alpha_{i}-r=k \alpha_{i}-\beta_{i}=\beta_{i+1}$ [by (b)]. It is easy to choose the end vertices of these edges in $V_{i+1}$ [using (c) and (d)] in such a way that the indegrees in $V_{i+1}$ differ by at most 1 . This proves the claim.

It is obvious that the diameter of the resulting graph is at least $D$. To prove that it is at most $D$, we note that all indegrees and all outdegrees are positive (with trivial exceptions for $V_{0}$ and $V_{D}$ ). This follows from (c) and the above construction. Now any two vertices of $G$ are connected by a path of length at most $D$, either through $V_{0}$ or through $V_{D}$. This completes the proof.

We remark that the conditions in Lemma 4 are redundant. For example, from (a), (c), and (d), it follows that all $\alpha_{i}$ and $\beta_{i}$ are positive. However, it was not our objective to give an economic set of conditions. All conditions, superfluous or not, are easy to check where we apply the lemma.

\section{LOWER BOUNDS ON $n_{0}(k, D)$}

Using Lemma 3, we obtain the following lower bounds on $n_{0}(k, D)$ in case $D \geq 4$. For $D<4$, we obtain exact values for $n_{0}(k, D)$ in Section 5 .

\section{Theorem 5.}

(a) If $D \geq 4$, then

$$
\begin{aligned}
& n_{0}(k, D) \geq \frac{k}{2} \cdot D \\
& + \begin{cases}2 k & \text { if } D \equiv 0(\bmod 4) \\
\frac{3}{2} k+2 & \text { if } D \equiv 1(\bmod 4) \\
k+4 & \text { if } D \equiv 2(\bmod 4) \\
\frac{k}{2}+2(\lceil 2 \sqrt{k}\rceil-1) & \text { if } D \equiv 3(\bmod 4) .\end{cases}
\end{aligned}
$$

(b) If $D \geq 10, D \equiv 2(\bmod 4)$, and $k$ is odd, then

$$
n_{0}(k, D) \geq \frac{k}{2} \cdot D+k+6 .
$$

Proof. (a) Let $D \geq 4$. Suppose that $G$ is a $(k, D)$-graph and let $f_{1}, f_{2}, \ldots, f_{D}$ be defined as in Section 2. Then, $n_{0}(k$, $D)=2 \sum_{i=1}^{D} f_{i}$, and $f_{1}=1, f_{2}=k-1$, and $f_{D-1}+f_{D}$ $\geq k$. Let $D=4 q+r$ with $0 \leq r \leq 3$.

If $r=0$, using Lemma $3(\mathrm{a})$, we obtain $\sum_{i=3}^{D-2} f_{i}$ $\geq(q-1) k$; hence, $n_{0}(k, D) \geq 2(q-1) k+4 k$ $=(k / 2) \cdot D+2 k$.

If $r=1$, we similarly obtain (using $\left.f_{i} \geq 1\right) n_{0}(k$, $D) \geq 2(q-1) k+4 k+2=(k / 2) \cdot D+(3 / 2) k$ +2 , and if $r=2$, we obtain $n_{0}(k, D) \geq 2(q-1) k$ $+4 k+4=(k / 2) \cdot D+k+4$.

If $r=3$, using Lemma 3(a) and (c), we obtain $\sum_{i=3}^{D-5} f_{i} \geq(q-1) k$ and $f_{D-4}+f_{D-3}+f_{D-2} \geq\lceil 2 \sqrt{k}\rceil$ -1 ; hence, $n_{0}(k, D) \geq 2(q-1) k+2(\lceil 2 \sqrt{k}\rceil-1)$ $+4 k=(k / 2) \cdot D+(k / 2)+2(\lceil 2 \sqrt{k}\rceil-1)$.

(b) Suppose that $f_{D-2}=f_{D-3}=1$ and $f_{D-7}+f_{D-6}+f_{D-5}$ $+f_{D-4}=k$. Then, as in the proof of Lemma 3(b) using Lemma $1(\mathrm{c})$, we obtain from $k f_{D-6} \leq\left(f_{D-7}\right.$ $\left.+f_{D-6}\right)\left(f_{D-6}+f_{D-5}\right)$ and $k f_{D-5} \leq\left(f_{D-6}+f_{D-5}\right)\left(f_{D-5}\right.$ $\left.+f_{D-4}\right)$ that $f_{D-6} f_{D-4}=f_{D-7} f_{D-5}$. Similarly, $k f_{D-4}$ $\leq\left(f_{D-5}+f_{D-4}\right)\left(f_{D-4}+f_{D-3}\right)$ implies that $\left(f_{D-7}\right.$ $\left.+f_{D-6}\right) f_{D-4} \leq\left(f_{D-5}+f_{D-4}\right) f_{D-3}$. Together with $f_{D-6} f_{D-4}=f_{D-7} f_{D-5}$, this implies that $\left(f_{D-4}+f_{D-5}\right) f_{D-7}$ $\leq\left(f_{D-4}+f_{D-5}\right) f_{D-3} \leq f_{D-4}+f_{D-5}$, so that $f_{D-7}=1, f_{D-5}$ $=f_{D-6} f_{D-4}$, and $k=f_{D-7}+f_{D-6}+f_{D-5}+f_{D-4}=(1$ $\left.+f_{D-6}\right)\left(1+f_{D-4}\right)$. Now from $k=k f_{D-3} \leq\left(f_{D-4}\right.$ $\left.+f_{D-3}\right)\left(f_{D-3}+f_{D-2}\right)=\left(f_{D-4}+1\right) 2$, we obtain $f_{D-6}$ $=1$ and $f_{D-5}=f_{D-4}=(k / 2)-1$. In particular, we obtain that $k$ is even. This means that for odd $k$ we can increase the lower bound for $n_{0}(k, D)$ in Theorem 5 (a) by 2 .

Theorem 6. If $D \geq 4$ and $k$ is prime, then

$$
\begin{aligned}
& n_{0}(k, D) \geq \frac{k+1}{2} \cdot D \text { if } D \equiv 0(\bmod 4) \\
&+ \begin{cases}2 k-2 & \text { if } D \equiv 1(\bmod 4) \\
\frac{3}{2} k-\frac{1}{2} & \text { if } D \equiv 2(\bmod 4) \\
k+1 & \text { if } D \equiv 3(\bmod 4) \\
\frac{k}{2}+2(\lceil 2 \sqrt{k}\rceil-1)-\frac{7}{2}\end{cases}
\end{aligned}
$$

Proof. Using Lemma 3(b) instead of Lemma 3(a), it is easy to prove Theorem 6 using the same arguments as in the proof of Theorem $5(\mathrm{a})$. We leave the details to the reader.

\section{UPPER BOUNDS ON $n_{0}(k, D)$}

Using Lemma 4 and suitable constructions, we obtain the following upper bounds on $n_{0}(k, D)$ in the case $D \geq 8$ 
and $k \geq 4$. For $D<8$ or $k<4$, we obtain exact values for $n_{0}(k, D)$ in Section 5 .

Theorem 7. Let $u, v, w, x$, and $k$ be positive integers such that $k=u+v+w+x$ and $u w=v x$. If $D=4 q+r \geq 8$ with $r \in\{0,1,2,3\}$, then

$$
n_{0}(k, D) \leq \frac{k}{2} \cdot D+ \begin{cases}2 k & \text { if } r=0 \\ \frac{3}{2} k+2 u & \text { if } r=1 \\ k+2(u+v) & \text { if } r=2 \\ \frac{k}{2}+2(u+v+w) & \text { if } r=3 .\end{cases}
$$

Proof. First suppose that $D=8$. Consider the $f$-sequence and the corresponding $a$-sequence below:

$$
\begin{aligned}
& f: 1,(k-1), u, v, w, x,(k-1), 1 \\
& a: 1, k,(k+u-1),(u+v),(v+w),(w+x),(x \\
& \quad+k-1), k, 1 .
\end{aligned}
$$

It is clear that the $a$-sequence and the $b$-sequence corresponding to the $f$-sequence (recall that $b_{i}=k f_{i}, i=1,2$, $\ldots, D)$ satisfy conditions (a) and (b) of Lemma 4 . To check (c) of Lemma 4, note that for $i \geq 1, a_{i} \leq(k-1)$ $+f_{i} \leq k f_{i}=b_{i}$, and for $3 \leq i \leq D-2, b_{i}=k f_{i} \leq k f_{i}+(k$ $\left.-\left(f_{i}+f_{i+1}\right)\right) f_{i+1}=k f_{i}+k f_{i+1}-\left(f_{i}+f_{i+1}\right) f_{i+1} \leq(k-1)\left(f_{i}\right.$ $\left.+f_{i+1}\right)=(k-1) a_{i}$. The remaining cases $(i=1,2, D$ -1 ) are easy to check. Condition (d) of Lemma 4 can be checked as follows: $b_{4}=k v=u v+v^{2}+v w+v x=u v$ $+v^{2}+v w+u w=(u+v)(v+w)=a_{3} a_{4} ; b_{5}$ runs similarly, and the remaining cases are even simpler.

Hence, there exists a $(k, D)$-graph corresponding to the indicated $f$-sequence. Repeating the subsequence $u$, $v, w, x$, adding an extra $u$, an extra $u$, or an extra $u$, $v, w$ at the end of the last subsequence $u, v, w, x$ in the case $r=1,2$, or 3 , respectively, and using similar arguments as above, one easily shows that there exists a ( $k$, $D$ )-graph with $D=4 q+r \geq 8$ on $n$ vertices, where $n=2$ $\sum_{i=1}^{D} f_{i}$. Using $k=u+v+w+x$, we get $2\left(\sum_{i=1}^{4 q-2} f_{i}\right.$ $\left.+f_{D-1}+f_{D}\right)=2(2 k+(q-1) k)=(k / 2) \cdot D+(2-(r /$ 2)) $k$. From this we obtain

$$
n_{0}(k, D) \leq \frac{k}{2} \cdot D+ \begin{cases}2 k & \text { if } r=0 \\ \frac{3}{2} k+2 u & \text { if } r=1 \\ k+2(u+v) & \text { if } r=2 \\ \frac{k}{2}+2(u+v+w) & \text { if } r=3\end{cases}
$$

Corollary 8. If $D \geq 8, k \geq 4$, and $k$ is even, then

$$
n_{0}(k, D) \leq \frac{k}{2} \cdot D+ \begin{cases}2 k & \text { if } D \equiv 0(\bmod 4) \\ \frac{3}{2} k+2 & \text { if } D \equiv 1(\bmod 4) \\ k+4 & \text { if } D \equiv 2(\bmod 4) \\ \frac{3}{2} k+2 & \text { if } D \equiv 3(\bmod 4) .\end{cases}
$$

Proof. Apply Theorem 7 with $u=v=1$ and $w=x$ $=(k / 2)-1$.

Corollary 9. If $D \geq 8, k \geq 5$, and $k$ is a nonprime odd number, then

$$
\begin{aligned}
n_{0}(k, D) \leq & \frac{k}{2} \cdot D \\
& + \begin{cases}2 k & \text { if } D \equiv 0(\bmod 4) \\
\frac{3}{2} k+2 & \text { if } D \equiv 1(\bmod 4) \\
k+2 d & \text { if } D \equiv 2(\bmod 4) \\
\frac{k}{2}+2\left(d^{\prime}+d^{\prime \prime}-1\right) & \text { if } D \equiv 3(\bmod 4)\end{cases}
\end{aligned}
$$

where $d$ is the smallest odd divisor $\geq 3$ of $k$, and $d^{\prime}$ and $d^{\prime \prime}$ are divisors of $k$ such that $k=d^{\prime} d^{\prime \prime}$ and $d^{\prime}+d^{\prime \prime}$ is minimum.

Proof. Apply Theorem 7 with $u=1, v=d-1, w$ $=(d-1)[(k / d)-1]$, and $x=(k / d)-1$ if $D \neq 3(\bmod$ $4)$, and with $u=d^{\prime}-1, v=1, w=d^{\prime \prime}-1$, and $x=\left(d^{\prime}\right.$ $-1)\left(d^{\prime \prime}-1\right)$ if $D \equiv 3(\bmod 4)$.

For prime numbers $k$, it is not possible to find a partition satisfying the hypothesis of Theorem 7 , in accordance with the lower bounds on $n_{0}(k, D)$ in Theorem 6.

Theorem 10. If $D \geq 8, k \geq 5$, and $k$ is prime, then

$$
n_{0}(k, D) \leq \frac{k+1}{2} \cdot D+ \begin{cases}2 k-2 & \text { if } D \equiv 0(\bmod 4) \\ \frac{3}{2} k-\frac{1}{2} & \text { if } D \equiv 1(\bmod 4) \\ k+1 & \text { if } D \equiv 2(\bmod 4) \\ \frac{5}{2} k-\frac{7}{2} & \text { if } D \equiv 3(\bmod 4)\end{cases}
$$


Proof. The proof is similar to the proof of Theorem 7 and Corollary 8 , starting with the $f$-sequence indicated below for $D=8$ :

$$
f: 1,(k-1), 1,1, \frac{k-1}{2}, \frac{k-1}{2},(k-1), 1 .
$$

We leave the details to the reader.

Note that the upper bounds in Theorem 10 hold for any odd $k \geq 5$. In the case $D \equiv 2(\bmod 4)$, these bounds could be better than those in Corollary 9 .

For fixed $k$, the upper bounds found so far make a relatively large jump when going from $D \equiv 2(\bmod 4)$ to $D \equiv 3(\bmod 4)$. This can be smoothed out by giving a better upper bound in the case $D \equiv 3(\bmod 4)$.

Theorem 11. Let $k \geq 4$ and $D=4 q+7$ with $q \geq 1$. Then,

$$
n_{0}(k, D) \leq \frac{k+e(k)}{2} \cdot D+\frac{k}{2}+4\lceil\sqrt{k}\rceil-2-\frac{7}{2} e(k),
$$

where $e(k)$

$$
= \begin{cases}0 & \text { if } k \text { is a square, } \\ 1 & \text { if } k \text { is not a square and } k \geq\lceil\sqrt{k}\rceil(\lceil\sqrt{k}\rceil-1), \\ 2 & \text { otherwise. }\end{cases}
$$

Proof. Consider the following $f$-sequence: $1,(k-1)$, $(\lceil\sqrt{k}\rceil-1), 1,(\lceil\sqrt{k}\rceil-1),(k+e(k)-2\lceil\sqrt{k}\rceil+1), \ldots$, $(\lceil\sqrt{k}\rceil-1), 1,(\lceil\sqrt{k}\rceil-1),(k-1), 1$, where the subsequence $f_{3}, f_{4}, f_{5}, f_{6}$ is repeated $q$ times.

If $k$ is a square [so $e(k)=0$ ], using Theorem 7 with $u=w=\sqrt{k}-1$ and $v=1, x=k+1-2 \sqrt{k}$, we obtain the result. If $k$ is not a square and $k \geq\lceil\sqrt{k}\rceil(\lceil\sqrt{k}\rceil-1)$ [ so $e(k)=1$ ], we are in a similar situation as in Theorem 10 (the sum of the elements $f_{3}, \ldots, f_{6}$ is $k+1$ ). Using Lemma 4 , it is not difficult to complete the proof for this case. In the last case, using $k \geq(\lceil\sqrt{k}\rceil-1)^{2}+1$ and Lemma 4 , it is again not difficult to complete the proof. We leave the details to the reader.

\section{EXACT VALUES OF $n_{0}(k, D)$}

In this section, we first determine $n_{0}(k, D)$ for $D=2$, $\ldots, 7$ and $k \geq 2$.

Theorem 12. Let $k \geq 2$.

(a) $n_{0}(k, 2)=2 k$,

(b) $n_{0}(k, 3)=2 k+2$,

(c) $n_{0}(k, 4)=4 k$ (d) $n_{0}(k, 5)=4 k+2$,

(e) $n_{0}(k, 6)=4 k+4$,

(f) $n_{0}(k, 7)=4 k+2\lceil 2 \sqrt{k}\rceil-2(k \geq 3)$.

Proof.

(a) The only ( $k, 2)$-graph is $K_{k, k}$.

(b) Let $V_{i}, a_{i}(i=0,1,2,3)$, and $b_{i}(i=1,2,3)$ be defined as in Section 2. Lemma 1 (d) gives $a_{0}+a_{2}$ $=a_{1}+a_{3}$. Now, $a_{3} \geq 1$; hence, $n_{0}(k, 3) \geq 2\left(a_{1}\right.$ $\left.+a_{3}\right) \geq 2 k+2$. The value $2 k+2$ is realized by the graph " $K_{k+1, k+1}$ minus a perfect matching."

(c) From Theorems 5 and 6 , we obtain $n_{0}(k, 4) \geq 4 k$. This lower bound can easily be attained. When $k$ $=2$, we simply take $C_{8}$; when $k \geq 3$, let $G$ be a ( $k$ $-1,3$ )-graph on $2 k$ vertices [cf. (b)]. Then, $K_{2} \times G$ satisfies all conditions.

(d) From Theorems 5 and 6, we obtain $n_{0}(k, 5) \geq 4 k$ +2 . The sequences $\left(a_{0}, \ldots, a_{5}\right)=(1, k, k, k, k, 1)$ and $\left(b_{1}, \ldots, b_{5}\right)=\left(k, k^{2}-k, k, k^{2}-k, k\right)$ satisfy the conditions of Lemma 4 ; hence, the value $4 k+2$ can be realized.

(e) From Theorems 5 and 6, we obtain $n_{0}(k, 6) \geq 4 k$ +4 . The sequences $\left(a_{0}, \ldots, a_{6}\right)=(1, k, k, 2, k, k$, $1)$ and $\left(b_{1}, \ldots, b_{6}\right)=\left(k, k^{2}-k, k, k, k^{2}-k, k\right)$ satisfy the conditions of Lemma 4 ; hence, the value $4 k+4$ can be realized.

(f) From Theorems 5 and 6 , we obtain $n_{0}(k, 7) \geq 4 k$ $+2\lceil 2 \sqrt{k}\rceil-2$. The following constructions show that equality holds if $k \geq 3$. If $k>\lceil\sqrt{k}\rceil(\lceil\sqrt{k}\rceil-1)$, consider the following a-sequence: $1, k,(k+\lceil\sqrt{k}\rceil-2)$, $\lceil\sqrt{k}\rceil,\lceil\sqrt{k}\rceil,(k+\lceil\sqrt{k}\rceil-2), k, 1$, and $b$-sequence: $k$, $(k-1) k_{2}(\lceil\sqrt{k}\rceil-1) k, k,(\lceil\sqrt{k}\rceil-1) k,(k-1) k, k$. If $k \leq\lceil\sqrt{k}\rceil(\lceil\sqrt{k}\rceil-1)$, then consider the following $a$ sequence: $1, k,(k+\lceil\sqrt{k}\rceil-2),\lceil\sqrt{k}\rceil,\lceil\sqrt{k}\rceil-1,(k+$ $\lceil\sqrt{k}\rceil-3), k, 1$, and $b$-sequence: $k,(k-1) k,(\lceil\sqrt{k}\rceil$ $-1) k, k,(\lceil\sqrt{k}\rceil-2) k,(k-1) k, k$. Except for $k$ $=2$, these sequences satisfy the conditions of Lemma 4.

Next, we determine $n_{0}(k, D)$ for $k=2,3,4$.

\section{Theorem 13.}

(a) $n_{0}(2, D)=2 D(D \geq 2)$,

(b) $n_{0}(3, D)=2 D+4(D \geq 4)$,

(c) $n_{0}(4, D)=2 D+8(D \geq 4)$.

Proof.

(a) The cycles are the only connected 2-regular graphs. 
(b) For $D \in\{4,5,6,7\}$, see Theorem 12 . Let $D \geq 8$. If $D$ is even, then $n_{0}=2\left(a_{1}+a_{3}+\cdots+a_{D-1}\right)$ $\geq 2 \cdot(3+[(D-4) / 2] \cdot 2+3)$ since $a_{3}, a_{5}, \ldots$, $a_{D-3} \geq 2$. If $D$ is odd, then $n_{0}=2\left(a_{0}+a_{2}+\cdots\right.$ $\left.+a_{D-1}\right) \geq 2 \cdot(1+3+[(D-5) / 2] \cdot 2+3)$ for similar reasons. Hence, $n_{0}(3, D) \geq 2 D+4$. Now choose $a_{0}$ $=a_{D}=1, a_{1}=a_{2}=k, a_{3}=\cdots=a_{D-3}=2, a_{D-2}$ $=a_{D-1}=k$, and apply Lemma 4 .

(c) The proof is similar to the proof of $(b)$. We leave the details to the reader.

From here on, we let $D \geq 8$ and $k \geq 5$.

\section{Theorem 14.}

(a) If $D \equiv 0(\bmod 4)$, then

$$
n_{0}(k, D)= \begin{cases}\frac{k}{2} \cdot D+2 k & \text { if } k \text { is nonprime } \\ \frac{k+1}{2} \cdot D+2 k-2 & \text { if } k \text { is prime. }\end{cases}
$$

(b) If $D \equiv 1(\bmod 4)$, then

$$
\begin{aligned}
& n_{0}(k, D) \\
& = \begin{cases}\frac{k}{2} \cdot D+\frac{3}{2} k+2 & \text { if } k \text { is nonprime } \\
\frac{k+1}{2} \cdot D+\frac{3}{2} k-\frac{1}{2} & \text { if } k \text { is prime. }\end{cases}
\end{aligned}
$$

Proof. It turns out that for $D \equiv 0$ or $1(\bmod 4)$ the lower bounds of Theorems $5(\mathrm{a})$ and 6 coincide with the upper bounds of Corollaries 8 and 9 and Theorem 10 .

For the other residue classes of $D(\bmod 4)$, the situation is less satisfactory.

Theorem 15. If $D \equiv 2(\bmod 4)$, then

$$
n_{0}(k, D)= \begin{cases}\frac{k}{2} \cdot D+k+4 & \text { if } k \text { is even } \\ \frac{k}{2} \cdot D+k+6 & \text { if } k \text { is odd and } 3 \mid k \\ \frac{k+1}{2} \cdot D+k+1 & \text { if } k \text { is prime. }\end{cases}
$$

Proof. For $D \equiv 2(\bmod 4)$, and the values of $k$ indicated above, the lower bounds of Theorems $5(\mathrm{a})$ and (b) and 6 coincide with the upper bounds of Corollaries 8 and 9 and Theorem 10.

Theorem 16. If $D \equiv 3(\bmod 4)$, then

$$
n_{0}(k, D)= \begin{cases}\frac{k}{2} \cdot D+\frac{k}{2}+4 \sqrt{k}-2 & \text { if } k \text { is a square } \\ 3 D+11 & \text { if } k=6 \\ 4 D+10 & \text { if } k=7 \\ 4 D+14 & \text { if } k=8 \\ 5 D+17 & \text { if } k=10 .\end{cases}
$$

Proof. When $k$ is a square, the lower bound of Theorem 5 (a) coincides with the upper bound of Theorem 11 . For $k=6,8,10$, compare Theorem 5(a) and Corollary 8. For $k=7$, compare Theorems 6 and 11 .

Remark. An isolated case for which we have determined $n_{0}(k, D)$ is $k=5, D=11$ : The sequence $\left(a_{0}, \ldots, a_{11}\right)$ $=(1,5,5,2,3,4,4,3,2,5,5,1)$ shows that $n_{0}(5,11)$ $\leq 40$, whereas from Theorem 6 , we have $n_{0}(5,11) \geq 40$. The minimal cases for which we have not determined $n_{0}(k, D)$ are $(k, D)=(5,15),(11,11),(25,10)$.

\section{REFERENCES}

[1] J. C. Bermond, C. Delorme, and G. Farhi, Large graphs with given degree and diameter II. J. Combin. Theory $B$ 36 ( 1984 ) 32-48.

[2] J. A. Bondy and U. S. R. Murty, Graph Theory with Applications. Macmillan, London; Elsevier, New York (1976).

[3] H. J. Broersma and A. A. Jagers, The unique 4-regular graphs on 14 and 15 vertices with diameter 2. Ars Comb. 25C ( 1988) 55-62.

[4] C. Delorme, Large bipartite graphs with given degree and diameter. J. Graph Theory 8 ( 1985 ) 325-334.

[5] M. Fellows, P. Hell, and K. Seyffarth, Large planar graphs with given diameter and maximum degree. Disc. Appl. Math., to appear.

[6] M. Fellows, P. Hell, and K. Seyffarth, Constructions of dense planar networks. Submitted (prepript).

[7] M. C. Heydemann, Personal communication, 11th British Combinatorial Conference, London (1987).

Received February 11, 1995

Accepted April 18, 1995 